\title{
AMRH and the High Energy Reinicke Problem
}

\author{
A. I. Shestakov and J. A. Greenough
}

\section{May 14, 2001}

U.S. Department of Energy

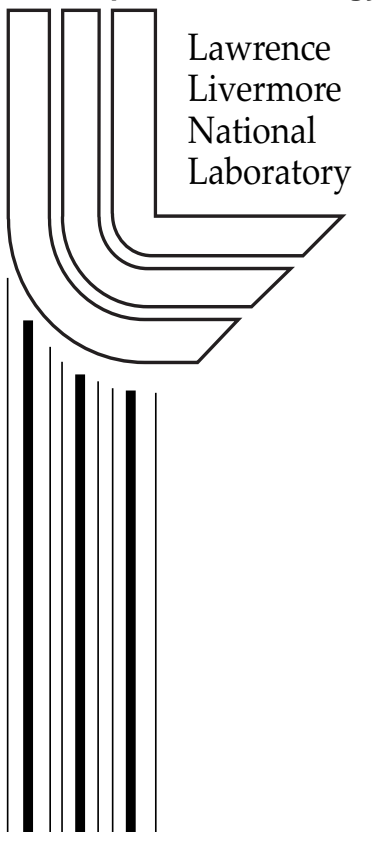




\section{DISCLAIMER}

This document was prepared as an account of work sponsored by an agency of the United States Government. Neither the United States Government nor the University of California nor any of their employees, makes any warranty, express or implied, or assumes any legal liability or responsibility for the accuracy, completeness, or usefulness of any information, apparatus, product, or process disclosed, or represents that its use would not infringe privately owned rights. Reference herein to any specific commercial product, process, or service by trade name, trademark, manufacturer, or otherwise, does not necessarily constitute or imply its endorsement, recommendation, or favoring by the United States Government or the University of California. The views and opinions of authors expressed herein do not necessarily state or reflect those of the United States Government or the University of California, and shall not be used for advertising or product endorsement purposes.

This work was performed under the auspices of the U. S. Department of Energy by the University of California, Lawrence Livermore National Laboratory under Contract No. W-7405-Eng-48.

This report has been reproduced directly from the best available copy.

Available electronically at http://www.doc.gov/bridge

Available for a processing fee to U.S. Department of Energy

And its contractors in paper from

U.S. Department of Energy

Office of Scientific and Technical Information

P.O. Box 62

Oak Ridge, TN 37831-0062

Telephone: (865) 576-8401

Facsimile: (865) 576-5728

E-mail: reports@adonis.osti.gov

Available for the sale to the public from

U.S. Department of Commerce

National Technical Information Service

5285 Port Royal Road

Springfield, VA 22161

Telephone: (800) 553-6847

Facsimile: (703) 605-6900

E-mail: orders@ntis.fedworld.gov

Online ordering: http://www.ntis.gov/ordering.htm

OR

Lawrence Livermore National Laboratory

Technical Information Department's Digital Library

http:/ / www.llnl.gov/tid/Library.html 


\title{
AMRH and the high energy Reinicke problem ${ }^{\dagger}$
}

\author{
A. I. Shestakov and J. A. Greenough \\ Lawrence Livermore National Laboratory \\ Livermore CA 94550 \\ E-mail: shestakov1@llnl.gov, greenough1@llnl.gov
}

We describe AMRH results on a version of the Reinicke problem specified by the V\&V group of LLNL's A-Div. The simulation models a point explosion with heat conduction. The problem specification requires that the heat conduction be replaced with diffusive radiation transport. The matter and radiation energy densities are tightly coupled.

\section{INTRODUCTION}

We present results obtained with the AMRH $\operatorname{code}^{1}$ on the high energy Reinicke problem, as specified by the Validation and Verification (V\&V) group of LLNL's A Division. Since the Reinicke problem has been previously described by Reinicke and Meyer-ter-Vehn (RMV) [1], Shestakov [2], and Shestakov et al [3], our introduction will be terse. Whenever possible, we use the same variable definitions as RMV and use CGS units. Our methodology for comparison, location of shock and thermal fronts, etc. is the same as [3], 22.1

As originally prescribed by RMV, the problem simulates a point explosion in an ideal gas. Thus, the EOS is

$$
p=(\gamma-1) \rho e=(\gamma-1) c_{\mathrm{v}} \rho T,
$$

where $\gamma$ is the specific heat ratio, $p$ denotes pressure, $\rho$ - density, $e$ - specific internal energy, $T$ - temperature, and $c_{\mathrm{v}}$ - the specific heat $\left(c_{\mathrm{v}}=\partial e / \partial T\right)$.

The gas motion is governed by the Euler equations for hydrodynamics enhanced by the addition of a diffusive flux of thermal energy. Specifically, the energy equation has the additional term: $-\nabla H$ where the heat flux

$$
H=-\chi_{0} \rho^{a} T^{b} \nabla T,
$$

and $\chi_{0}, a$, and $b$ are constants.

\footnotetext{
$\dagger$ This work was performed under the auspices of the U.S. Department of Energy by the University of California, Lawrence Livermore National Laboratory under contract number W-7405ENG-48.

${ }^{1}$ In Apr. '01, RAPTOR and AMRH were the names of the code and project respectively.
} 
Because some codes do not have heat conduction packages, the V\&V group modified RMV's original formulation and modeled heat conduction using radiation diffusion. To this end, the term $-\nabla H$ is replaced by the radiation-to-matter coupling term

$$
\mathcal{K}=c \rho \kappa_{P}\left(B-E_{r}\right) \text {, }
$$

where $c$ is the speed of light, $\kappa_{P}$ is a coupling parameter, $E_{r}$ is the radiation energy density, and the integrated Planck function,

$$
B=(4 \sigma / c) T^{4}
$$

where $\sigma$ is the Stefan-Boltzmann constant. The radiation field evolves per,

$$
\partial_{t} E_{r}=\nabla D_{r} \nabla E_{r}+\mathcal{K}
$$

where the radiation diffusion coefficient,

$$
D_{r}=c / 3 \kappa_{R}
$$

The above prescription reduces to RMV's original formulation. First, consider the matter energy balance equation,

$$
\rho \partial_{t} e=\rho c_{\mathrm{v}} \partial_{t} T=-\mathcal{K}
$$

Next, assume tight coupling between matter and radiation and a power law prescription for the radiation mean-free-path, i.e.,

$$
\kappa_{P} \gg 1 \text { and } \kappa_{R}=\kappa_{R, 0} \rho^{m} T^{-n}
$$

where $\kappa_{R, 0}, m$, and $n$ are constants. In this limit, $E_{r} \rightarrow B$. Hence, using (1), (2) and (4) reduce to

$$
\partial_{t} E_{r}+\rho c_{\mathrm{v}} \partial_{t} T=\nabla D^{\prime} \nabla T
$$

where $D^{\prime}=\left(16 \sigma / 3 \kappa_{R, 0}\right) \rho^{-m} T^{n+3}$. On the left-side of (5), using CGS units, the first term is approximately $7.57 \cdot 10^{-15} \partial_{t}\left(T^{4}\right)$. Thus, if $\rho=c_{\mathrm{V}}=\mathcal{O}(1)$, and if $T$ is not too large, the first term may be neglected leaving the desired expression,

$$
\rho c_{\mathrm{v}} \partial_{t} T=\nabla D^{\prime} \nabla T=\nabla\left(\chi_{0} \rho^{a} T^{b} \nabla T\right)
$$

where $\chi_{0}=\left(16 \sigma / 3 \kappa_{R, 0}\right), a=-m$, and $b=n+3$. We emphasize that the assumption $T=\mathcal{O}(1)$, which allowed us to neglect the first term on the left-side of $(5)$, is violated in the initial stages of the simulation at small $r$, since at $t=0, T \propto \delta(r)$. Thus, only after an initial transient should we expect the numerical solution to approach the one derived by RMV.

We use parameter settings conveyed by J. Bolstad [4] of the V\&V group. The initial density satisfies,

$$
\left.\rho\right|_{t=0}=g_{0} r^{\kappa}
$$


where $g_{0}$ and $\kappa$ are constants. The initial energy - all internal - is concentrated at the center of a sphere,

$$
(\rho E)_{t=0}=(\rho e)_{t=0}=\mathcal{E}_{0} \delta(r) .
$$

Outside of the central region, the gas is cold, i.e., $p=T=0$. The remaining constants are,

$$
\gamma=7 / 5, \quad c_{\mathrm{v}}=1 /(\gamma-1), a=-2, b=6, g_{0}=\chi_{0}=1 .
$$

The problem is then completely determined once $\kappa$ and $\mathcal{E}_{0}$ are specified.

RMV show that if $\kappa$ is given by,

$$
\kappa=(1-6 b) /(2 b-2 a+1),
$$

then, the problem may be redefined as a system of ODEs for the reduced (nondimensional) variables,

$$
\begin{aligned}
\xi & =r /\left(\zeta t^{\alpha}\right) \\
U(\xi) & =u(r, t) /(\alpha r / t) \\
\Theta(\xi) & =\Gamma T(r, t) /(\alpha r / t)^{2} \\
G(\xi) & =\rho(r, t) /\left(g_{0} r^{\kappa}\right),
\end{aligned}
$$

where the constant $\zeta$ depends on a certain integral of the reduced equations [1],

$$
\alpha=2 /(\kappa+5), \Gamma=c_{\mathrm{v}} /(\gamma-1),
$$

and $u$ is the physical velocity.

For comparison with integrations of the reduced variables, it is useful to combine the physical parameters into one nondimensional value

$$
\lambda=\left(2 \chi_{0} / \Gamma^{b+1} g_{0}^{1-a}\right) \cdot\left(\mathcal{E}_{0} / g_{0}\right)^{b-1 / 2}
$$

which characterizes the solution. For large $\lambda$, one obtains the "large $\mathcal{E}_{0}$ " or "supersonic" case which we have simulated. This regime is characterized by two expanding waves; a heat front at $r=r_{h}(t)$ ahead of a shock at $r=r_{s}(t)$. By self-similarity, both $r_{h}$ and $r_{s}$ evolve as $t^{\alpha}$. Thus, the constant ratio

$$
R=r_{h} / r_{s}
$$

is a metric which gauges the accuracy of the numerical solution.

Of course, $R$ is not the only characteristic of interest. The shape of $T(r)$ depends on the specific choice for $\gamma, a$, and $b$. For the set of values discussed in [1], [2], and [3], $\max (T)$ occurs at $r=0$. For the present V\&V specification, it arises at $r=r_{s}$.

Unfortunately, we do not know of an apriori way to compute $R$ for a given set of physical parameters. Previous work [2] and [3], by trial and error, obtained parameter settings yielding $R \approx 2.0$ and for those settings $\lambda=3.3685 \cdot 10^{14}$ For the settings $(6)$, in order to also obtain $R \approx 2$, we use,

$$
\mathcal{E}_{0}=135.0
$$


which yields,

$$
\lambda=1.042 \cdot 10^{12}
$$

\section{NUMERICAL RESULTS}

We now present results from two AMRH simulations. Both discretize the domain $0 \leq r \leq 1.0$ into $N$ uniform cells. The simulations use $N=256$ and 512. The simulations run to

$$
t=t_{\max }=0.06
$$

Figure 1 displays the profiles at the final time. By inspection, we find $r_{h} \approx 0.98$ and $r_{s} \approx 0.47$. Qualitatively, the agreement between the $N=256$ and 512 simulations is good, although there are noticeable differences: The front $r_{h}$ for $N=512$ is slightly further out and at $r=0, T_{512}<T_{256}$. Since the densities are in good agreement and since $T \propto e$, this is in line with the expectation that the ratio of total internal energy to the total energy,

$$
R_{\mathcal{E}} \doteq\left(\int \rho e d V\right) / \mathcal{E}_{0}
$$

is approximately the same. For our simulations

$$
R_{\mathcal{E}}=0.63565,0.63873
$$

for $N=256,512$ resp.

Besides the differences in $T$, Fig. 1 shows that the densities generally agree, expect for their maxima; $\max \left(\rho_{256}\right)$ is $3.8 \%$ lower than the corresponding maximum for $N=512$. The other maxima are in closer agreement; $\max \left(u_{256}\right)$ is within $0.3 \%$ of $\max \left(u_{512}\right)$ and $\max \left(T_{256}\right)$ is within $0.5 \%$ of $\max \left(T_{512}\right)$. However, taking a cue from the behavior of $\rho$ for the original RMV parameters ([3], §2.1), we should not expect good agreement since $\rho$ is so peaked at $r=r_{s}$.

Another noticeable feature in Fig. 1 is the "step" in $u$ and $\rho$ at $r \approx 0.21$ for the $N=256$ case. To a lesser extent, this error persists for $N=512$ and is evidenced by the slight wave in the $u$ profile at $r \approx 0.29$. This glitch is a remnant of the early transient, and goes away as the solution is refined, or, for a fixed mesh size, as the problem evolves and more points are enveloped by the outgoing waves. As a side comment, the early transient is unphysical. At early time, $\rho$ has two local maxima; one at $r=0$ and another at $r=r_{s}$. What happens is that $\rho(t=0)$, which monotonically decreases away from the origin, must "flip over" to resemble the self-similar result in which $\rho$ monotonically increases away from $r=0$. This transition occurs gradually. When the "shock" first forms, it arises a few cells away from $r=0$ and at that time, $\max (\rho)$ still occurs at the origin.

We now display plots evidencing the self-similarity of the numerical solution. Because of the early transients, it makes no sense to analyze the solution too early in time. One must wait for numerical self-similarity to establish itself. We note that the waiting time depends on the resolution. If properly scaled, the numerical solution with $h=1 / 256$ is nearly identical to the one with $h=1 / 512$. Proper scaling requires that the time steps evolve "proportionate" time steps, e.g., limiting 


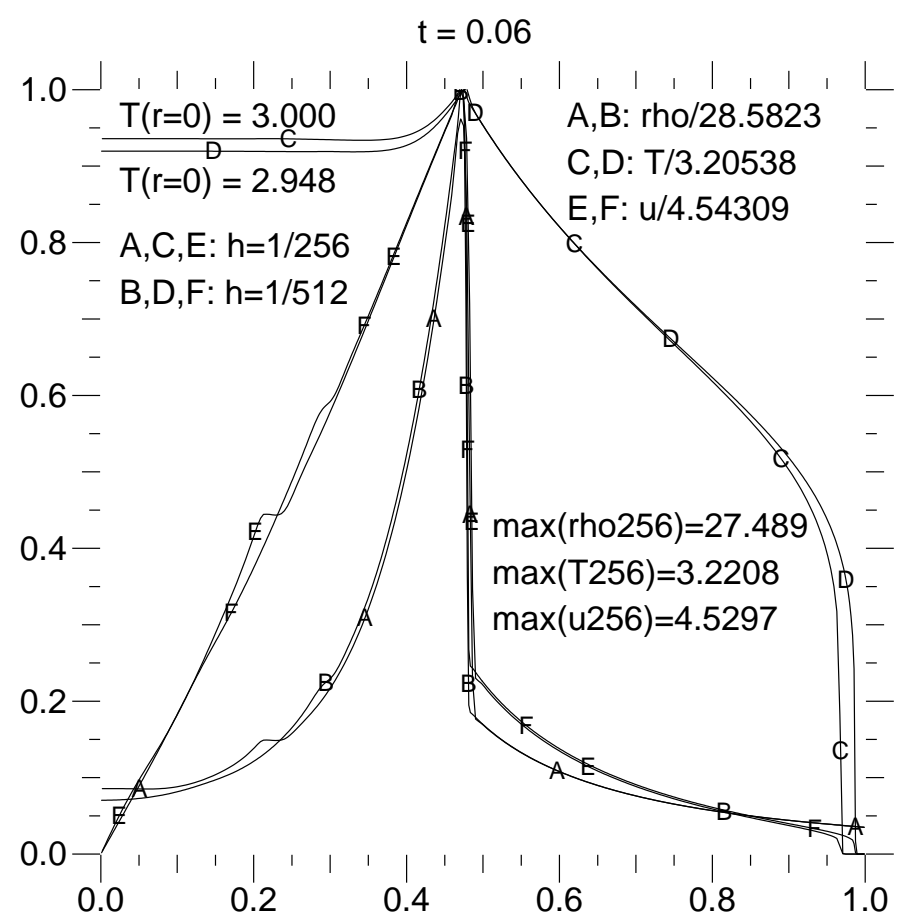

FIG. 1. Line plots of $\rho, T$, and $u$ at final time. Curves are normalized to the respective maxima of the results for $N=512$.

$\Delta t$ with a small Courant number, and comparing when the two solutions have enveloped the same number of cells.

Figure 2 which displays the ratio $R$ - see (11) - shows that for $N=512, R$ appears to be stabilizing at $R \approx 2.08$ The $N=256$ case appears to be still increasing at $t=t_{\mathrm{max}}$; the run concludes with $R \approx 2.045$ yielding approximately a $2 \%$ discrepancy. One should not expect too much accuracy in the definition of $R$ since it depends on how $r_{s}$ and $r_{h}$ are defined. Numerically, these values are subject to interpretation errors of $\mathcal{O}(h)$, see [3], 2.1

We next compute three quantities that display self-similarity of the numerical solution. Recalling (8), (9), and (10), we plot $\max (u, T, \rho)$ taking care to scale the variables in order to obtain values that should be time-independent. Figures 3, 4, and 5 resp. display the scaled velocity, temperature, and density. For the scaling, the variables are evaluated at $r=r_{s}$ which we define as the position where $\rho$ attains its maximum.

Figures 3, 4, and 5 further attest to the establishment of self-similarity, albeit showing how long it takes for it to develop. For example, although Figs. 3 and 4 imply that by $t=0.03$ and $N=512$, both $u$ and $T$ have attained self-similarity (since the scaled maxima vary only in the third decimal digit), Fig. 5 implies that even at $t=0.06$, the scaled maximum of $\rho$ still varies its second digit. However, care should be exercised before judging. Since, $\rho$ is so highly peaked at $r=r_{s}$, it takes a long time before we can trust more than two digits of $\max (\rho)$.

Lastly, we derive an estimate for $\zeta$ - see (7). First, using the Fig. 2 result, $R=2.08$, and recalling RMV's convention that $\xi=1$ at $r=r_{s},(7)$ shows that 


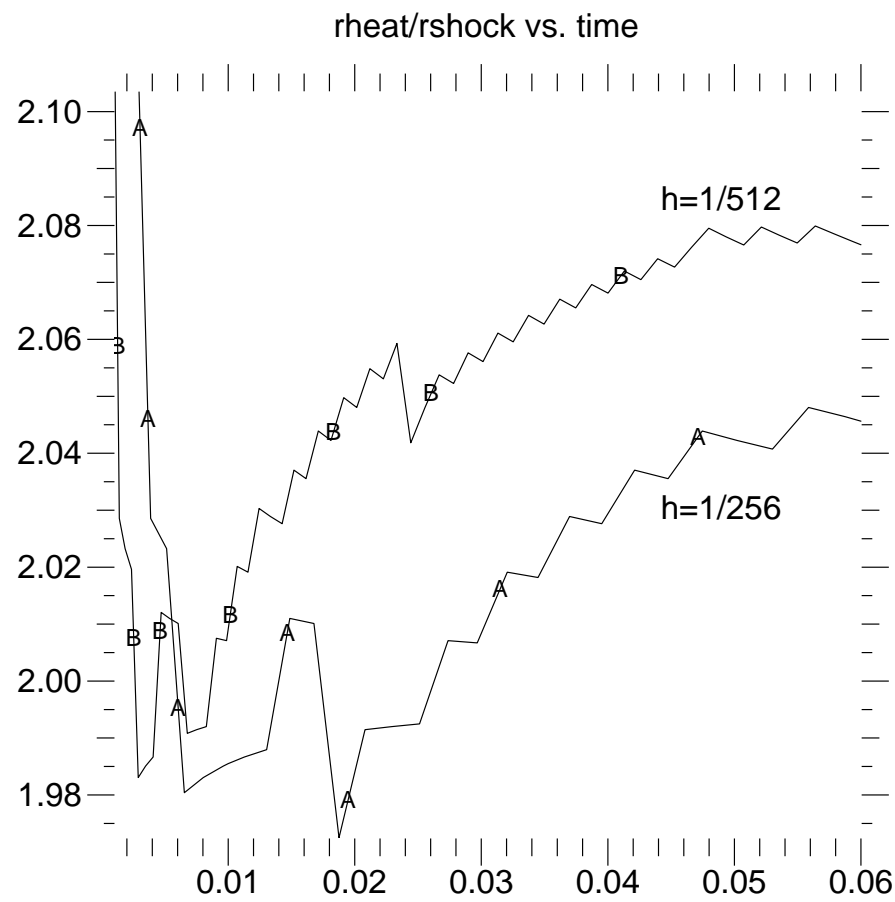

FIG. 2. Ratio $R=r_{h} / r_{s}$ vs. $t$

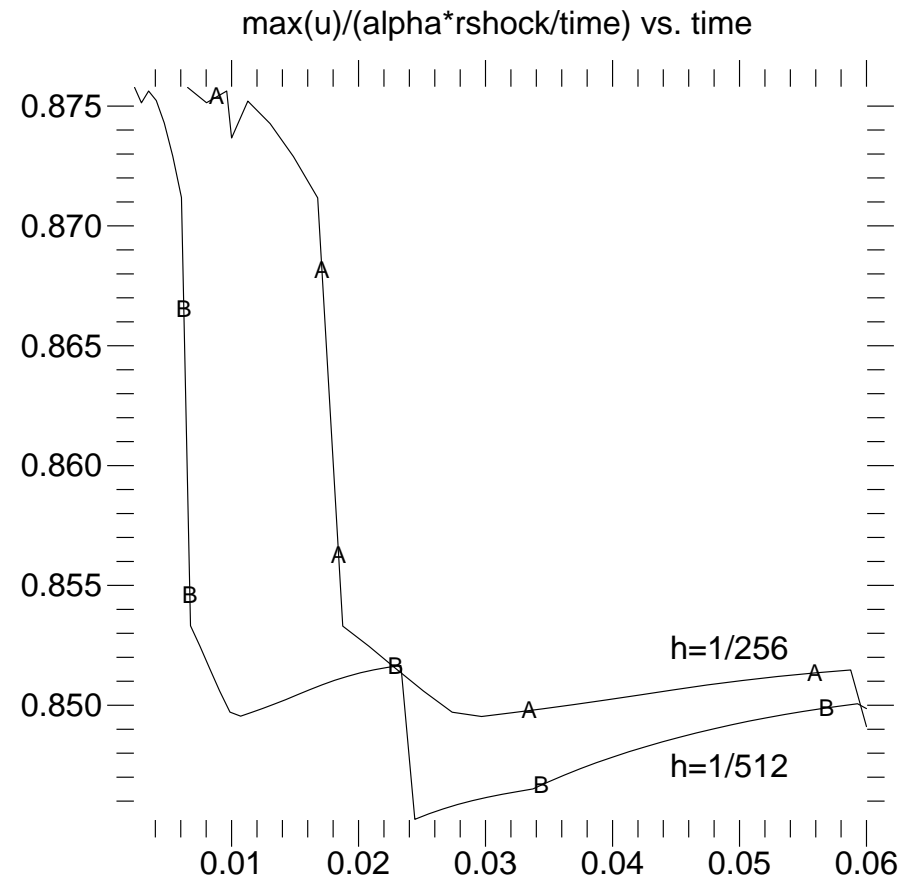

FIG. 3. Normalized maximum velocity $\max (u) /\left(\alpha r_{s} / t\right)$ vs. $t$. 


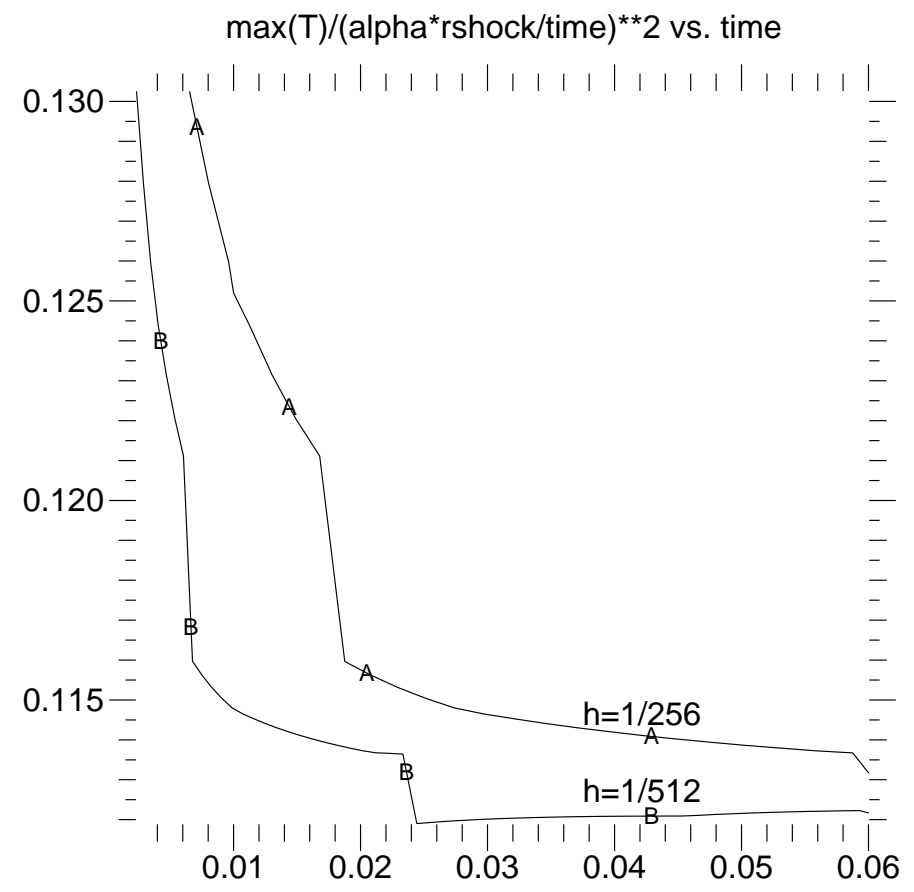

FIG. 4. Normalized maximum temperature $\max (T) /\left(\alpha r_{s} / t\right)^{2}$ vs. $t$.

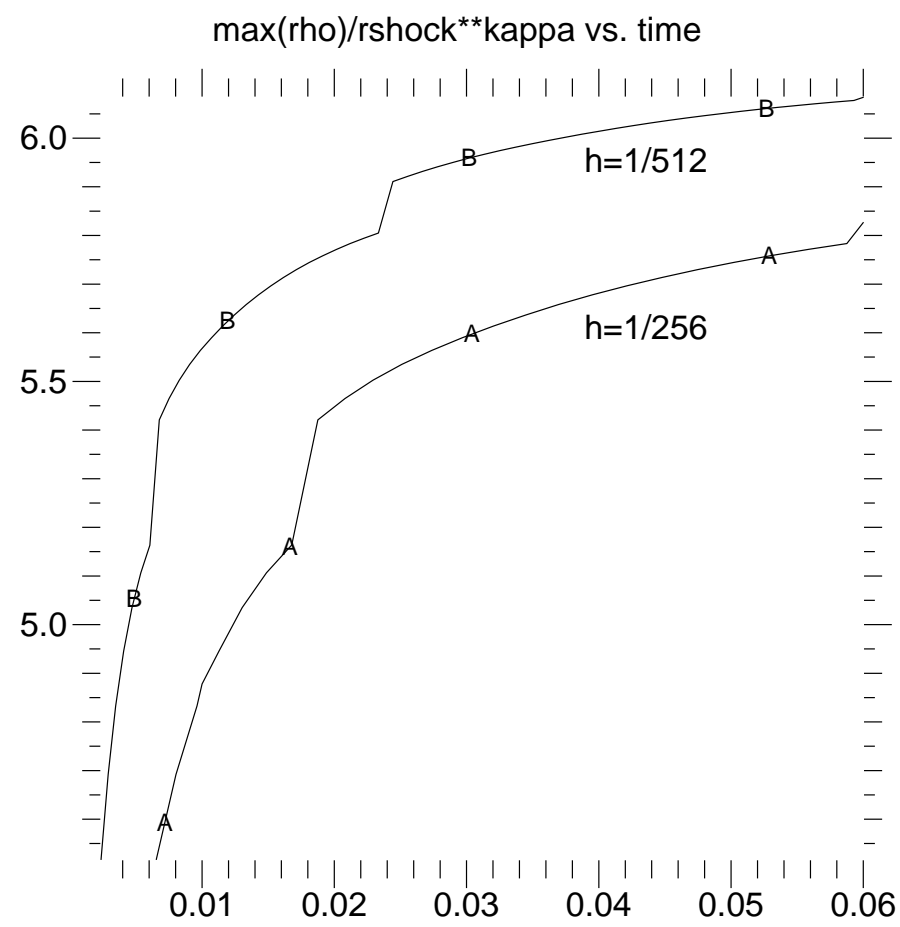

FIG. 5. Normalized maximum density $\max (\rho) / r_{s}^{\kappa}$ vs. $t$. 
$\xi=R=2.08$ at $r=r_{h}$. This in turn yields,

$$
\zeta=3.19
$$

\section{CONCLUSION}

Using the AMRH code in $1 \mathrm{D}$, spherical coordinates, we have simulated a highenergy Reinicke problem for two discretizations. We demonstrated that as time evolves, the numerical solution approaches self-similarity. The simulation was run using the code's hydrodynamic module and radiation diffusion modules. The latter, developed by L. Howell of LLNL [5], was run in backward Euler, fully implicit mode. The radiation module, which couples the radiation diffusion and matter energy balance equations, for each time cycle, solves a nonlinear system of equations. The module solves for the radiation energy density $E_{r}$ and specific internal energy $e$. For this problem, because of the ideal gas EOS, $e \sim T$. Thus, the nonlinearities are due to the dependencies $B \propto T^{4}$ and $\kappa_{R} \propto T^{-n}$. The code uses CGS units. For the simulation, we set the coupling parameter $\kappa_{P}=10^{10}$. For the Rosseland opacity $\kappa_{R}$, defined in (4), we used $\kappa_{R, 0}=16 \sigma / 3, m=2$, and $n=3$.

\section{REFERENCES}

1. P. Reinicke and J. Meyer-ter-Vehn, Phys. Fluids A, 3 (7), (1991) p. 1807

2. A. I. Shestakov, "Time-dependent simulations of point explosions with heat conduction," Phys. Fluids, 11, 5, May 1999, p. 1091

3. A. I. Shestakov, J. L. Milovich, and M. K. Prasad, "Combining Cell and Point Centered Methods in 3D, Unstructured-Grid Radiation-Hydrodynamic Codes," to appear in J. Comp. Phys., and available as Lawrence Livermore National Laboratory Report, UCRL-JC-137456, Rev. 1, Livermore CA, Dec. 2000

4. J. H. Bolstad, private communication, Lawrence Livermore National Laboratory, (2000)

5. L. H. Howell and J. A. Greenough, "A block-structured adaptive mesh refinement algorithm for diffusion radiation," Lawrence Livermore National Laboratory Report, UCRL-JC-133094, Livermore CA, 1998 